\title{
Mental health, disability and cardiovascular risks in South African adults with hypertension: structural equation models of causal pathways
}

Max Oscar Bachmann ( $\nabla$ m.bachmann@uea.ac.uk)

University of East Anglia Norwich Medical School https://orcid.org/0000-0003-1770-3506

\section{Arvin Bhana}

Centre for Rural Health, University of KwaZulu Natal School of Nursing and Public Health

Katherine Grady

University of East Anglia Norwich Medical School

Naomi Folb

Knowledge Translation Unit, University of Cape Town

Graham Thornicroft

Institute of Psychiatry, King's College London

Babalwa Zani

Knowledge Translation Unit, University of Cape Town

Crick Lund

Department of Psychiatry and Mental Health, University of Cape Town

\section{Carl Lombard}

Amref Health Africa

\section{Naomi Levitt}

Department of Diabetic Medicine and Endocrinology, University of Cape Town

\section{Lara Fairall}

Knowledge Translation Unit, University of Cape Town

Jill Hanass-Hancock

HIV Prevention Unit, South African Medical Research Council

Inge Petersen

Centre for Rural Health, University of Kwazulu Natal

\section{Research article}

Keywords: Depression, disability, multimorbidity, cardiovascular risk

Posted Date: March 11th, 2020

DOl: https://doi.org/10.21203/rs.3.rs-16834/v1

License: () (i) This work is licensed under a Creative Commons Attribution 4.0 International License. Read Full License 


\section{Abstract}

Aim South African adults with hypertension often have comorbid physical and mental health problems and disabilities, which can influence each other in complex ways. We modelled potential causal pathways connecting multimorbidity, depression, stress, disability and cardiovascular risks.

Methods The study analysed observational data on 1043 adults with treated hypertension and with symptoms of depression (Patient Health Questionnaire-9 score > 9) who participated in a randomised trial in 20 primary health care clinics. A structural equation path model using cross-sectional baseline data estimated direct and indirect effects of sociodemographic variables and comorbidities on depression, stress and disability scores, and their further direct and indirect effects on smoking, body mass index and blood pressure. A cross-lagged structural equation model using longitudinal data estimated effects of depression symptoms, disability and blood pressure on each other over time.

Results At baseline, depression symptoms as outcome were positively associated with previous myocardial infarction, blood pressure, and disability. Disability was positively associated with depression symptoms, stress scores, myocardial infarction and tuberculosis. Body mass index was positively associated with income and female sex and negatively associated with HIV, tuberculosis, perceived stress and age. Systolic blood pressure was positively associated with depression symptoms and age, and negatively associated with tuberculosis. Indirect effects of myocardial infarction and age on disability were partly mediated through perceived stress and depression symptoms. Baseline depression symptoms predicted greater disability after one year, and baseline disability predicted greater depression symptoms and higher blood pressure after one year.

Conclusions Control of cardiovascular risk factors in primary care patients with hypertension is interrelated with their mental health, disability, and comorbidity. Better mental health care and management of disability could help control blood pressure and reduce cardiovascular risk.

\section{Introduction}

Multimorbidity and depression are growing problems in low- and middle-income countries (LMICs) [1]. Adults with multiple physical conditions are more likely to report depressive episodes and anxiety symptoms[2, 3] and, in six LMICs including South Africa, depression and disability are associated with multimorbidity [4]. World Health Survey data show that comorbid depression and chronic diseases (angina, arthritis, asthma, diabetes) are associated with worse health compared with depression alone, and with any combination of these chronic diseases without depression [5].

The relationship between mental and physical conditions appears bidirectional [6]. Pre-existing anxiety and depression has been found to increase the risk of a wide range of chronic physical conditions [7], including myocardial infarction and stroke [8], and vice versa. Several studies have shown how disability and poor physical health impacts negatively on mental health. Kelley-Moore and Ferrarro [9] demonstrated that, in a United States cohort of older adults with depression, disability or disease at baseline, each factor independently predicted the presence of each of the other factors at later time points. Similarly, Ormel and colleagues showed that in a Netherlands cohort of older people with physical limitations, depressive symptoms and disability mutually reinforced each other over time [10]. Choi and colleagues found similar reciprocal relationships between depression and cardiovascular disease in a United States cohort [10].

While associations between physical and mental conditions are well established, the mechanisms linking physical and mental illness and disability are not fully understood, but are probably multifactorial, including social, psychological and pathophysiological factors [[6]. There is little evidence about these mechanisms in LMIC populations [1]. South Africa provides an ideal LMIC setting to study these relationships and mechanisms. It has high rates of communicable diseases such as HIV/AIDS and tuberculosis, as well as noncommunicable diseases (NCDs) $[12,13]$. Stroke, ischaemic heart disease, diabetes and hypertensive heart disease are the leading causes of NCD deaths in South Africa [14], for which hypertension and obesity are major risk factors. Further, South Africa has high prevalence of depression and anxiety $[15,16]$. The 2016 South African disability survey found that over half of adults aged 65 years and older reported some disability [17]. The high prevalence of multimorbidity, depression and disability in South Africans thus provides a suitable setting for investigating relationships between these conditions. 
We have previously investigated causal pathways linking socioeconomic factors and depression in South African adults with NCDs $[18,19]$. The present study goes on to investigate causal pathways linking physical multimorbidity, depression, stress, disability and cardiovascular risk factors in South African adults with hypertension. Data were collected during a randomised trial of a health system intervention intended to improve diagnosis and treatment of depression in patients with hypertension, and thereby also to improve blood pressure control, in public primary care facilities [20]. We analysed these longitudinal and cross-sectional data with the objectives of : 1) constructing structural equation models (SEMs) representing potential causal paths linking sociodemographic factors, multimorbidity, depression symptoms, stress scores, disability and cardiovascular risk factors; 2) identifying factors directly and indirectly associated with endogenous variables along these paths; and 3) investigating directions of effect between depression symptoms, disability and blood pressure.

\section{Methods}

The study design is a cross sectional and cohort study within a randomised trial. As part of an international programme to increase treatment of mental disorders in LMICs [21], the PRIME-SA cluster randomised controlled trial was conducted in the Dr Kenneth Kaunda district of the North West province of South Africa from 2015 to 2016 [20]. The largest 20 public sector primary care clinics in the district were included in the trial, with 10 clinics randomly allocated to continue with usual care and 10 to receive the intervention. Study participants were recruited from clinic waiting rooms and invited to volunteer participation, with screening for inclusion conducted in a private room. Inclusion criteria were receiving treatment for hypertension; depressive symptoms scoring nine or more on the Patient Health Questionnaire-9 (PHQ-9), which had been validated for the setting [22]; and age 18 years or older. Exclusion criteria were inability to provide informed consent, urgent need for medical attention and planning to move away during the 12-month follow-up period. The trial was conducted alongside the Comorbid Affective Disorders, AIDS/HIV, and Long Term Health (CobALT) trial, which recruited participants receiving antiretroviral therapy [23]. Participants receiving both antiretroviral therapy and antihypertensive medication were included in both trials and are included in the present study.

Participants in control arm clinics received usual care. This included training nurses in the use of an algorithm-based clinical management tool, which included guidelines for the detection and management of depression. This clinical management tool had been rolled out to the entire district by the Department of Health [24]). Patients were further supported for self-management of their condition by engagement with community health worker outreach teams. In intervention arm clinics, the PRIME-SA intervention was added to usual care and comprised: 1 ) a collaborative team-based model, with nurse practitioners as case managers; 2) additional training for nurse clinicians in identification and management of depression, and in clinical communication skills; 3) strengthening of referral pathways to primary care physicians for initiation of psychotropic medication; and 4) increased access to counselling in the clinics by lay behavioural health counsellors trained to provide individual and group-based counselling for patients with depressive symptoms. Access to referral to specialist mental health services was the same for both arms and was provided by two district-based psychologists, as well as psychologists based at the district and provincial psychiatric hospitals.

The planned sample size of 1000 (50 participants per clinic) was intended to provide $90 \%$ power to detect a $17 \%$ difference between arms in the primary outcome ( $\geq 50 \%$ reduction in PHQ-9 score after 6 months), assuming $15 \%$ loss to follow-up, intra-cluster correlation of 0.04 , and $5 \%$ significance level [20].

\section{Data collection}

Data were collected by interviewer-administered questionnaires using hand-held electronic devices at enrolment and after 12 months. The questionnaires included questions about age, current medication and depressive symptoms to assess for eligibility. In eligible and consenting participants, we collected additional data about sociodemographic details (age, sex, highest education level, employment, monthly income, marital status) self-reported comorbid conditions, depressive symptoms, perceived stress, and disability. Smoking status was recorded as a categorical variable and current smokers were asked about the number of cigarettes smoked per day. Data collectors took measurements for height, weight and blood pressure. Blood pressure was measured with a digital sphygmomanometer, with participants seated, at three time-points during the course of the interview. The first reading was discarded and the mean of the second two readings used to calculate a mean systolic and diastolic blood pressure. Body weight and height were measured to calculate body mass index (BMI) (weight/height $\left.{ }^{2}\right)$. 
Depressive symptoms were measured by the validated PHQ-9, a nine-item questionnaire asking participants how frequently they experienced various symptoms of depression over the last two weeks [25]. Responses were coded on a Likert scale as "0 days", "1-7 days", "8-11 days" and "12-14 days", with scores of 0, 1, 2 and 3, respectively. Scores for all nine items were added to produce a PHQ-9 depression score, with higher scores indicating more severe depression [22].

Disability was measured with the 12-item World Health Organization Disability Assessment Scale 2.0 (WHODAS 2.0), which is suitable for use with mental health disorders and is linked to the concepts in the International Classification of Functioning, Disability and Health [26]. It covers six domains of functioning: cognition, mobility, self-care, interaction with others, life activities and participation in community activities. Participants were asked to rate their level of difficulty with activities of functioning on a fivepoint scale, with 0 representing no difficulty and 4 representing extreme difficulty or inability to perform that activity. Scores for each item were weighted using the WHODAS 2.0 complex scoring method and added to produce a disability score.

The degree to which situations in a person's life are appraised as stressful was measured by the 10-item Perceived Stress Measures scale [27]. The items ask how unpredictable, uncontrollable and overloaded respondents find their lives. Frequencies are expressed as "never", "almost never", "sometimes", "fairly often" and "very often".

Data collectors were trained to administer the questionnaire and take measurements. Alerts were imbedded in the questionnaire to flag participants at risk, and we conducted quality control to check and correct unusual readings.

\section{Statistical Analysis}

Distributions of categorical variables were summarised with percentages, and distributions of continuous variables were summarised with means and standard distributions. We estimated Cronbach's alpha inter-item correlation for PHQ-9, Perceived Stress and WHODAS 2.0 scores. We investigated crude associations between explanatory variables and depression symptoms, stress and disability scores with linear regression models, and also estimated Pearson correlation coefficients when both paired variables were continuous.

We constructed two structural equation path models (SEMs) representing potential causal pathways linking exogenous sociodemographic variables and comorbidities, and endogenous depression, stress and disability scores, and cardiovascular risk factors. For analysis of cross-sectional data, we assumed that depression and stress scores were primarily causes, not effects, of disability (Fig. 1), and tested this assumption with further longitudinal analysis described below. This SEM was a series of linear regression models, comprising successive endogenous outcome variables and their respective cumulative explanatory variables. The exogenous variables of education, employment, diabetes and stroke were removed from the model because they were found not to be independently associated with any endogenous variable in the SEM.

Regression coefficients were interpreted as controlled direct effects, that is, as effects not mediated through intermediate variables between exposure and outcome. All endogenous variables were continuous, so indirect or mediated effects were estimated by multiplication of coefficients from relevant regression models. Indirect effects were expressed as the total indirect effects of an exposure on an outcome, mediated through all mediators between them.

For analysis of longitudinal data, we constructed a cross-lagged SEM, to investigate whether depression, disability and blood pressure were causes or effects of each other (Fig. 2). The model is similar to that of Ormel and colleagues who investigated bidirectional effects of depression and disability over time (Ormel et al, 2002). Follow-up measurements of depression score, disability score and systolic blood pressure were the outcomes and were adjusted for baseline values of the same variable, while baseline measurements of the other variables were covariates representing possible causes. This regression method avoids regression to the mean due to correlation between variables at baseline and ensures that supposed causes were measured before supposed effects. The model also adjusted for baseline values of age, sex, marital status, myocardial infarction, tuberculosis, and HIV, which were potential confounders. Potential effects of the intervention were accounted for by including a covariate indicating intervention versus control arm but were not the focus of the present study. We removed perceived stress from a more complex crosslagged model after finding no evidence of bidirectional effects between stress and either disability or blood pressure. Indirect effects were not estimated with the primary cross-lagged model, which had no mediating variables (Fig. 2). However, additional mediation analyses were carried out to investigate whether the effect of baseline disability on blood pressure at follow-up was mediated through BMI, and whether effects of blood pressure at baseline on disability at follow-up was mediated through myocardial infarction or stroke. The latter mediation analyses used Stata's user-written package "medeff". 
For both SEMs, continuous variables were standardised so that the magnitude of effects of different variables on different outcomes could be compared with each other. All SEMs used robust variance correction of errors for intra-cluster correlation of outcomes between clinics. Goodness of fit of SEMs with the data was expressed as equation level and overall $\mathrm{R}^{2}$ values. Likelihood ratio test statistics, information criteria and root mean square errors of approximation could not be estimated together with robust adjustment for clustering, and so were estimated after repeating the SEM analyses without robust adjustment.

To assess the assumption that outcomes variables were normally distributed we firstly plotted histograms of each variable, and of residuals predicted by each regression model. To assess the sensitivity of regression results to deviations from normality we repeated the SEM analyses using non-parametric bootstrapping, randomly sampling clusters with replacement, with 1000 iterations, and estimated standard errors and confidence intervals with the bias corrected accelerated method.

Statistical analysis was carried out using STATA version 15.0. A 5\% significance level was used.

\section{Results}

At baseline, $82 \%$ of participants were women and $82 \%$ were aged 40 years or older (Tables 1 and 2). Educational, employment and income levels were low, with $73 \%$ of participants never having been to secondary school, $26 \%$ unemployed, and mean monthly income Rand 1200 (about US\$84 in 2016). Multimorbidity was common, with 29\% receiving treatment for HIV, 19\% ever having had a myocardial infarction and $19 \%$ ever having had tuberculosis. Forty six percent were obese $\left(\mathrm{BMI}>30 \mathrm{~kg} / \mathrm{m}^{2}\right), 46 \%$ had systolic blood pressure above $140 \mathrm{mmHg}$, indicating poor control, and 13\% currently smoked. 
Table 1

Participants' characteristics and associations with depression, stress and disability scores at baseline

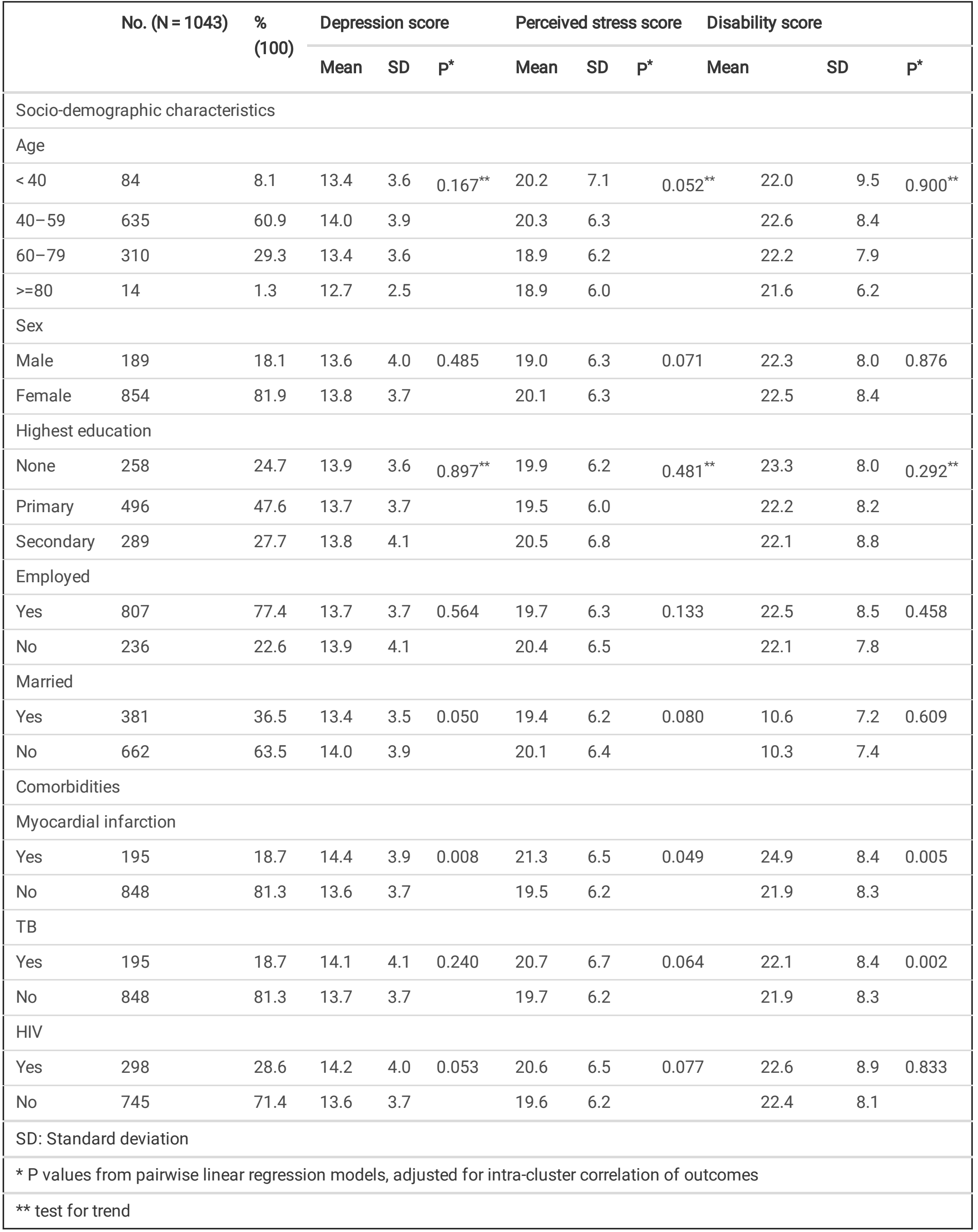


Table 2

Distribution of continuous variables and inter-variable correlations at baseline $(\mathrm{N}=1043)$

\begin{tabular}{|c|c|c|c|c|c|c|c|c|c|c|}
\hline & \multirow[t]{2}{*}{ Mean } & \multirow[t]{2}{*}{ SD } & \multicolumn{8}{|c|}{ Correlation (Pearson correlation coefficients below diagonal and $\mathrm{P}$ values above it) } \\
\hline & & & Age & Income & Depression & $\begin{array}{l}\text { Perceived } \\
\text { Stress }\end{array}$ & Disability & Cigarettes/day & BMI & SBP \\
\hline Age (years) & 54.3 & 10.7 & & 0.034 & 0.167 & 0.052 & 0.900 & 0.678 & 0.459 & $\begin{array}{l}<.001 \\
0.0\end{array}$ \\
\hline $\begin{array}{l}\text { Monthly } \\
\text { income } \\
\text { (thousand } \\
\text { Rand) }\end{array}$ & 1.20 & 1.43 & 0.074 & & 0.673 & 0.453 & 0.820 & 0.398 & 0.125 & 0.891 \\
\hline $\begin{array}{l}\text { Depression } \\
\text { score }\end{array}$ & 16.8 & 3.8 & -0.040 & 0.013 & & $<0.001$ & $<0.001$ & 0.152 & 0.702 & 0.026 \\
\hline $\begin{array}{l}\text { Perceived } \\
\text { Stress score }\end{array}$ & 19.9 & 6.3 & -0.106 & -0.020 & 0.314 & & $<0.001$ & 0.089 & 0.230 & 0.420 \\
\hline $\begin{array}{l}\text { Disability } \\
\text { score }\end{array}$ & 22.4 & 8.4 & 0.005 & -0.007 & 0.295 & 0.410 & & 0.238 & 0.933 & 0.257 \\
\hline Cigarettes/day & 0.13 & 0.34 & -0.006 & -0.022 & 0.049 & 0.065 & 0.040 & & $\begin{array}{l}< \\
0.001\end{array}$ & 0.094 \\
\hline $\begin{array}{l}\text { Body mass } \\
\text { index }\left(\mathrm{kg} / \mathrm{m}^{2}\right)\end{array}$ & 30.1 & 8.6 & -0.025 & 0.044 & -0.015 & -0.043 & -0.004 & -0.234 & & 0.678 \\
\hline $\begin{array}{l}\text { Systolic blood } \\
\text { pressure (mm } \\
\mathrm{Hg})\end{array}$ & 141.5 & 25.4 & 0.210 & -0.003 & 0.083 & 0.032 & 0.0538 & 0.062 & -0.011 & \\
\hline
\end{tabular}

At baseline, scores for depressive symptoms were statistically significantly higher in participants who were unmarried, reported myocardial infarction or had higher systolic blood pressure (Tables 1 and 2). Perceived stress scores were significantly higher in participants who reported myocardial infarction. Disability scores were significantly higher in participants who reported myocardial infarction or previous tuberculosis. Depression, perceived stress and disability scores were all positively and significantly associated each other, with correlation coefficients between 0.295 and 0.410 (Table 2). Cronbach's alpha coefficients for the PHQ-9, perceived stress and disability scores were $0.46,0.76$ and 0.83 , respectively, at baseline and $0.81,0.83$ and 0.85 at follow-up.

The SEM of baseline data estimated direct and indirect effects on endogenous variables along the causal paths modelled (Fig. 1), with adjustment for confounding by covariates (Table 3, Fig. 3). Higher depression score as outcome was independently and directly associated with myocardial infarction, as was higher perceived stress scores, which was also associated with younger age. Higher disability score as outcome was independently and directly associated with higher depression and perceived stress scores, and with older age, myocardial infarction and tuberculosis. Higher disability score was indirectly associated with younger age and myocardial infarction, mediated through their effects on depression and perceived stress (Table 3). Higher BMI as an outcome was independently and directly associated with female sex, higher income and myocardial infarction, and was inversely associated with higher perceived stress score, smoking, tuberculosis and HIV. Younger age was indirectly associated with higher BMI, mediated through its effect on perceived stress (Table 3). Finally, higher systolic blood pressure as an outcome was independently and directly associated with higher depression score, older age, and not having had tuberculosis. Secondary analysis, using non-parametric bootstrap to assess the sensitivity of these results to departures from the assumed normal distributions of outcomes, produced similar results (Supplementary Table 1). Direct effects identified in the primary analysis remained statistically significant (that is, $95 \%$ confidence intervals excluded zero) with bootstrap estimation, the only exceptions being effect of age on perceived stress, and effect of sex on BMI, which became non-significant. 
Table 3

Structural equation model: direct and indirect effects at baseline

\begin{tabular}{|c|c|c|c|c|c|c|}
\hline \multirow[t]{2}{*}{ Outcome \& explanatory variables } & \multicolumn{3}{|c|}{ Direct effects } & \multicolumn{3}{|c|}{ Indirect effects } \\
\hline & Coefficient & $95 \% \mathrm{Cl}$ & $\mathrm{P}$ & Coefficient & $95 \% \mathrm{Cl}$ & $P$ \\
\hline \multicolumn{7}{|l|}{ Outcome: $\mathrm{PHQ}$ score $\left(\mathrm{R}^{2}=0.019\right)$} \\
\hline Age & -0.027 & $-0.079,0.025$ & 0.306 & & & \\
\hline Sex (female) & 0.046 & $-0.105,0.196$ & 0.552 & & & \\
\hline Married & -0.106 & $-0.252,0.039$ & 0.153 & & & \\
\hline Monthly income & 0.026 & $-0.032,0.084$ & 0.376 & & & \\
\hline Myocardial infarction & 0.237 & $0.091,0.382$ & 0.001 & & & \\
\hline Tuberculosis & 0.044 & $-0.116,0.205$ & 0.588 & & & \\
\hline HIV & 0.144 & $-0.037,0.325$ & 0.119 & & & \\
\hline \multicolumn{7}{|l|}{ Outcome: Stress score $\left(R^{2}=0.033\right)$} \\
\hline Age & -0.094 & $-0.187,0.000$ & 0.049 & & & \\
\hline Sex (female) & 0.147 & $-0.020,0.313$ & 0.085 & & & \\
\hline Married & -0.054 & $-0.174,0.065$ & 0.374 & & & \\
\hline Monthly income & $<0.001$ & $-0.053,0.053$ & 0.991 & & & \\
\hline Myocardial infarction & 0.306 & $0.040,0.572$ & 0.024 & & & \\
\hline Tuberculosis & 0.087 & $-0.054,0.229$ & 0.227 & & & \\
\hline HIV & 0.087 & $-0.090,0.264$ & 0.334 & & & \\
\hline \multicolumn{7}{|c|}{ Outcome: Disability score $\left(\mathrm{R}^{2}=0.178\right)$} \\
\hline PHQ score & 0.187 & $0.093,0.276$ & $<0.001$ & & & \\
\hline Stress score & 0.347 & $0.272,0.405$ & $<0.001$ & & & \\
\hline Age & 0.042 & $0.001,0.097$ & 0.048 & -0.037 & $-0.068,-0.005$ & 0.023 \\
\hline Sex (female) & -0.020 & $-0.219,0.170$ & 0.808 & 0.058 & $-0.010,0.126$ & 0.094 \\
\hline Married & 0.121 & $-0.028,0.220$ & 0.129 & -0.038 & $-0.094,0.018$ & 0.184 \\
\hline Monthly income & -0.006 & $-0.070,0.067$ & 0.971 & 0.005 & $-0.017,0.027$ & 0.661 \\
\hline Myocardial infarction & 0.208 & $0.035,0.363$ & 0.017 & 0.147 & $0.046,0.248$ & 0.004 \\
\hline Tuberculosis & 0.208 & $0.081,0.341$ & 0.001 & 0.038 & $-0.029,0.105$ & 0.267 \\
\hline HIV & -0.056 & $-0.199,0.077$ & 0.385 & 0.056 & $-0.031,0.143$ & 0.208 \\
\hline \multicolumn{7}{|c|}{ Outcome: Cigarettes smoked per day $\left(R^{2}=0.061\right)$} \\
\hline PHQ score & 0.027 & $-0.033,0.086$ & 0.375 & 0.013 & $-0.005,0.032$ & 0.165 \\
\hline Stress score & 0.013 & $-0.031,0.058$ & 0.557 & 0.025 & $-0.009,0.058$ & 0.146 \\
\hline Disability score & 0.071 & $-0.026,0.171$ & 0.148 & & & \\
\hline Age & -0.043 & $-0.098,0.011$ & 0.121 & -0.001 & $-0.009,0.006$ & 0.772 \\
\hline Sex (female) & -0.577 & $-0.839,-0.314$ & $<0.001$ & 0.006 & $-0.013,0.025$ & 0.556 \\
\hline
\end{tabular}




\begin{tabular}{|c|c|c|c|c|c|c|}
\hline \multirow{2}{*}{$\begin{array}{l}\text { Outcome \& explanatory variables } \\
\text { Married }\end{array}$} & \multicolumn{3}{|c|}{ Direct effects } & \multicolumn{3}{|c|}{ Indirect effects } \\
\hline & -0.100 & $-0.298,0.102$ & 0.338 & 0.001 & $-0.016,0.018$ & 0.945 \\
\hline Monthly income & 0.053 & $-0.074,0.180$ & 0.416 & 0.001 & $-0.005,0.007$ & 0.740 \\
\hline Myocardial infarction & -0.038 & $-0.128,0.052$ & 0.409 & 0.036 & $-0.005,0.076$ & 0.083 \\
\hline Tuberculosis & -0.006 & $-0.182,0.170$ & 0.944 & 0.020 & $-0.002,0.042$ & 0.068 \\
\hline HIV & -0.077 & $-0.256,0.103$ & 0.406 & 0.005 & $-0.015,0.024$ & 0.639 \\
\hline \multicolumn{7}{|c|}{ Outcome: Body mass index $\left(R^{2}=0.198\right)$} \\
\hline PHQ score & 0.009 & $-0.056,0.079$ & 0.742 & 0.001 & $-0.013,0.015$ & 0.877 \\
\hline Stress score & -0.065 & $-0.106,-0.015$ & 0.009 & 0.005 & $-0.020,0.030$ & 0.706 \\
\hline Disability score & 0.035 & $-0.050,0.095$ & 0.538 & -0.006 & $-0.013,0.002$ & 0.152 \\
\hline No. cig. smoked & -0.077 & $-0.138,-0.014$ & 0.016 & & & \\
\hline Age & -0.086 & $-0.138,-0.032$ & 0.002 & 0.009 & $0.003,0.015$ & 0.005 \\
\hline Sex (female) & 0.781 & $0.636,0.926$ & $<0.001$ & 0.036 & $-0.003,0.075$ & 0.070 \\
\hline Married & 0.080 & $-0.026,0.190$ & 0.137 & 0.011 & $-0.005,0.026$ & 0.176 \\
\hline Monthly income & 0.071 & $0.005,0.136$ & 0.034 & -0.004 & $-0.011,0.004$ & 0.314 \\
\hline Myocardial infarction & 0.041 & $-0.080,0.167$ & 0.487 & -0.008 & $-0.043,0.028$ & 0.667 \\
\hline Tuberculosis & -0.339 & $-0.437,-0.236$ & $<0.001$ & $<0.001$ & $-0.019,0.019$ & 0.982 \\
\hline HIV & -0.484 & $-0.613,-0.357$ & $<0.001$ & 0.002 & $-0.013,0.016$ & 0.817 \\
\hline \multicolumn{7}{|c|}{ Outcome: Systolic blood pressure $\left(R^{2}=0.054\right)$} \\
\hline PHQ score & 0.070 & $0.004,0.137$ & 0.038 & 0.004 & $-0.016,0.023$ & 0.715 \\
\hline Stress score & 0.050 & $-0.005,0.108$ & 0.072 & 0.005 & $-0.030,0.040$ & 0.780 \\
\hline Disability score & 0.015 & $-0.092,0.114$ & 0.837 & 0.003 & $-0.005,0.011$ & 0.477 \\
\hline No. cig. smoked & 0.038 & $-0.054,0.132$ & 0.416 & $<0.001$ & $-0.005,0.004$ & 0.923 \\
\hline Body mass index & 0.003 & $-0.056,0.061$ & 0.922 & & & \\
\hline Age & 0.194 & $0.133,0.254$ & $<0.001$ & -0.009 & $-0.018,0.001$ & 0.083 \\
\hline Sex (female) & 0.132 & $-0.270,0.006$ & 0.061 & -0.009 & $-0.083,0.066$ & 0.823 \\
\hline Married & 0.024 & $-0.097,0.146$ & 0.688 & -0.013 & $-0.032,0.006$ & 0.181 \\
\hline Monthly income & -0.031 & $-0.073,0.011$ & 0.147 & 0.004 & $-0.002,0.011$ & 0.217 \\
\hline Myocardial infarction & 0.024 & $-0.154,0.203$ & 0.787 & 0.036 & $-0.017,0.089$ & 0.181 \\
\hline Tuberculosis & -0.168 & $-0.283,-0.051$ & 0.005 & 0.010 & $-0.023,0.043$ & 0.553 \\
\hline HIV & 0.028 & $-0.115,0.170$ & 0.704 & 0.010 & $-0.030,0.051$ & 0.617 \\
\hline
\end{tabular}


Table 4

Associations between baseline characteristics and depression, disability and systolic blood pressure at follow-up: cross lag structural equation model

\section{Outcomes and explanatory variables}

Coefficient

$95 \%$ confidence interval

$\mathbf{P}$

Outcome: Depression score at follow-up $\left(R^{2}=0.116\right)$

Disability score at baseline

0.225

$0.148,0.302$

$<0.001$

Systolic blood pressure at baseline

0.058

Depression score at baseline

0.164

$-0.017,0.134$

0.072

Age

$-0.003$

$0.094,0.235$

$<0.001$

Sex (female)

0.055

$-0.066,0.059$

0.920

Married

$-0.047$

$-0.135,0.245$

0.503

Myocardial infarction

$-0.011$

Tuberculosis

0.164

HIV

$-0.132$

Intervention vs control arm

$-0.066$

$-0.203,0.109$

0.474

0.894

$-0.190,0.169$

0.052

$-0.002,0.330$

0.076

Outcome: Disability score at follow-up $\left(R^{2}=0.136\right)$

Depression score at baseline

0.092

$-0.254,-0.010$

0.722

Systolic blood pressure at baseline

0.086

$-0.430,0.298$

Disability score at baseline

0.260

Age

0.096

$0.020,0.164$

0.021

Sex (female)

0.260

$0.005,0.167$

0.007

Married

0.111

$0.170,0.351$

$<0.001$

Myocardial infarction

0.050

Tuberculosis

0.078

HIV

$-0.011$

Intervention vs control arm

$-0.142$

$0.005,0.187$

0.003

$0.122,0.398$

0.001

$-0.017,0.238$

0.088

0.535

$-0.111,0.210$

0.352

$-0.113,0.268$

0.880

$-0.193,0.171$

0.401

Outcome: Systolic blood pressure at follow-up $\left(R^{2}=0.275\right)$

Disability score at baseline

0.085

$-0.472,0.189$

Depression score at baseline

0.021

Systolic blood pressure at baseline

0.500

Age

0.024

$0.011,0.159$

0.005

Sex (female)

$-0.139$

$-0.060,0.101$

0.487

Married

$-0.036$

$-0.081$

Myocardial infarction

$-0.161$

Tuberculosis

0.066

HIV

$-0.030$

$0.402,0.599$

$<0.001$

$-0.036,0.085$

0.417

$-0.318,0.039$

0.062

$-0.133,0.062$

0.549

Intervention vs control arm

Page 10/16 
Goodness of fit: overall $\mathrm{R}^{2}=0.433$; likelihood ratio chi $^{2}$ test of model vs. saturated $=263(90 \% \mathrm{Cl} 0.275-0.338), \mathrm{df}=3, \mathrm{P}<0.0001$; root mean squared error of approximation $(\mathrm{RMSEA})=0.306$, probability RMSEA $<=0.05<0.001$; Bentler RMSEA $=0.290$; standardised root mean squared residual $=0.046$

The cross-lagged SEM of longitudinal data investigated directions of effect (Fig. 2, Supplementary appendix Table 1). It showed that baseline disability score was independently and most strongly associated with baseline-adjusted depression score at follow-up. Similarly, the baseline depression score was independently associated with baseline-adjusted disability score at follow-up. Baseline blood pressure was associated with baseline-adjusted disability score at follow-up, and vice versa, but baseline blood pressure was not independently associated with depression score at follow-up. No other covariates were independently associated with the three outcomes, except for older age and female sex which were associated with baseline-adjusted disability at follow-up. There were no statistically significant indirect effects of blood pressure on disability mediated through myocardial infarction or stroke, and no statistically significant indirect effects of disability on blood pressure mediated through BMI (results not shown).

\section{Discussion}

This study of South African primary care patients with hypertension provides insights into the complex and dynamic effects of sociodemographic factors, multimorbidity, depression symptoms, perceived stress and disability on each other and on cardiovascular risk factors. The results suggest that disability was a key variable in this web of causal paths, as a cause, effect and mediator of a variety of physical and mental health problems. Depression and disability appeared to have bidirectional effects on each other, with worse depression at baseline, associated with more disability at baseline and at follow-up, and vice versa (Fig. 3). This confirms similar evidence of bidirectional effects reported by Ormel and colleagues [10]. However the coefficient for disability as a predictor of depression (0.225) was larger than the coefficient for depression as a predictor of disability (0.092) - supporting our placement of disability before depression in the path model for baseline data (Fig. 1). Kelley-Moore and Ferraro also demonstrated a similar pattern regarding NCDs, disability and depression, with the presence of each factor at baseline predicting the others three years later [9]. In the present study, perceived stress was more strongly associated with disability at baseline than depression was, but we did not find equivalent evidence of bidirectional effects between stress and disability over time.

Most directly relevant to hypertension management were the findings that higher depression scores at baseline were directly associated with higher blood pressure at baseline and at follow-up, which could be due to poor treatment adherence among patients with more severe depression symptoms, or due to psycho-biological mechanisms. Longitudinal analysis suggested that disability and blood pressure also had bidirectional effects on each other. It is plausible that the effects of hypertension on disability could be mediated through cardiovascular disease and stroke, and that the effects of disability on blood pressure could be mediated through obesity or medication adherence, did not find evidence of such mediation.

These results, together with the finding that myocardial infarction was strongly associated with greater depression symptoms, are in keeping with previous studies showing associations between depression and cardiovascular disease. Several studies have shown depression to be associated with concurrent hypertension or cardiovascular disease and also to predict incident cardiovascular disease in the future $[6,8,27,28]$. A cohort study of United States Medicare beneficiaries aged 65 years or older, found bidirectional relationships between depression and cardiovascular disease over time [11].

In the present study, age and sex variables were not independently associated with depression symptoms, but older age was predictably associated with higher BMI, systolic blood pressure and disability. However, because older age was associated with less perceived stress, it was indirectly associated with less disability at baseline, so that the direct and indirect of effects of age on disability cancelled each other out (Table 3 ). Females were much more likely than males to be obese ( $53 \%$ versus $18 \%$ having BMI > $30 \mathrm{~kg} / \mathrm{m}^{2}$ ) but female sex was not independently associated with any endogenous variable other than BMI.

Socioeconomic factors were generally not associated with differences in physical or mental health. Higher income was associated with higher BMI, possibly because it enabled purchase of more food. This finding contrasts with high income settings in which higher income is usually associated with less overweight and obesity [29]. Considering that $75 \%$ of participants earned less than US\$100 per month, this discrepancy could be because higher earners in our study were only relatively so, and could possibly only afford poor quality high calorie food. Education, employment and income had no other independent effects, which differs from our previous 
study which found all three variables to be independently associated with depression symptoms among South African primary care patients with long term conditions $[18,19]$. This difference between our studies may be partly because of the higher prevalence of poverty, and less socioeconomic variability, in the present study.

Comorbid physical conditions appeared to have a variety of adverse effects. Previous myocardial infarction was associated with higher depression, perceived stress and disability scores. The association between myocardial infarction and higher BMI is probably due to obesity and overweight causing myocardial infarction, but the reverse could also be true if myocardial infarction resulted in reduced physical activity. Tuberculosis was associated with disability but HIV was not, possibly because most people with HIV were well controlled on antiretroviral treatment ( $84 \%$ had undetectable viral loads at baseline). However, both tuberculosis and HIV were associated with lower BMI.

The study has several limitations. Firstly, although the SEMs represent supposed causal pathways, the associations could have been biased by unmeasured confounding factors. Secondly, the self-reported measures of depression, perceived stress and disability are inevitably prone to error. Self-reported comorbidities are also prone to error. Random measurement error in outcome measurement could bias associations between independent variables measured at baseline and changes in dependent variables, when modelled using baseline adjustment [30]. Alternative models using change score as outcome could avoid this bias, but could instead be biased by regression to the mean. That is, if baseline covariates are associated with baseline values of a variable, they would tend to be inversely associated with changes in that variable due to chance alone. Thirdly, the study population was restricted to participants with both depressive symptoms and a previous diagnosis of hypertension. This inclusion criterion of hypertension treatment and depressive symptoms could bias associations between depression symptoms and blood pressure, and means that these results should not be generalised to populations without both hypertension and depressive symptoms [30]. Fourthly, analyses of crosssectional baseline data were unable to identify directions of effects, but longitudinal data provided insights about directions of effects between depression, disability and blood pressure. Fifthly, one year of follow-up may be insufficient for gradual processes such as disability to develop, but there were several statistically significant predictors of changes in disability, depression score and blood pressure. Sixthly, outcome variables were not all normally distributed, as assumed for linear regression. Notably, baseline depression score was truncated at a lower limit of 9 , and most participants smoked zero cigarettes. However sensitivity analysis using bootstrapping supported the robustness of the primary analyses despite departures from normality. Finally, the cluster sample design reduced statistical power, so that robust adjustment for differences between clinics made many apparent associations nonsignificant, and precluded conventional goodness of fit estimates.

The study is original in its attempt to disentangle complex causal processes in a LMIC setting. The path models are arguably more informative than conventional regression models that simply pool diverse covariates without considering the mechanisms or directions of effect linking them. Inappropriate adjustment for mediating variables, or for covariates that are themselves affected by outcomes, can obscure effects. What is needed in future, to confirm or refute these exploratory findings, are larger longitudinal studies with longer follow-up in similar populations with high disease burdens in LMIC settings.

\section{Conclusions}

Given that depression has been found to be an important factor in the pathway to disability and poorer health outcomes in patients with NCDs, screening and interventions for depression in patients with chronic disease are needed. In particular, this study highlights the importance of targeted screening for the detection and treatment of depression in patients with hypertension, especially after myocardial infarction.

\section{Declarations}

\section{Ethics approval and consent to participate}

The authors assert that all procedures contributing to this work comply with the ethical standards of the relevant national and institutional committees on human experimentation and with the Helsinki Declaration of 1975, as revised in 2008. Ethical approval was obtained from the University of KwaZulu-Natal's Biomedical Research Ethics Committee (reference BFC049/15); the University of Cape Town's Faculty of Health Sciences Human Research Ethics Committee (reference HREC 412/2011) and the Department of 
Health in the North West Province of South Africa. Participants provided verbal consent for screening and written informed consent to participate in the trial before data collection began.

Consent for publication: Not applicable

\section{Availability of data and materials}

The anonymised participant-level data will be made publicly available 1 year after publication of the main trial outcome paper, in accordance with PRIME publication and data management policies.

\section{Competing interests}

The authors declare that they have no competing interests

\section{Funding}

This study is an output of the Programme for improving mental health care (PRIME). This work was supported by the United Kingdom Department for International Development (201446). GT is supported by the National Institute for Health Research (NIHR) Collaboration for Leadership in Applied Health Research and Care (CLAHRC) South London and by the NIHR Applied Research Centre (ARC) at King's College London NHS Foundation Trust, and the NIHR Applied Research and the NIHR Asset Global Health Unit award. The views expressed are those of the authors and not necessarily those of the NHS, the NIHR or the Department of Health and Social Care. GT receives support from the National Institute of Mental Health of the National Institutes of Health under award number R01MH100470 (Cobalt study). GT is supported by the UK Medical Research Council in relation the Emilia (MR/S001255/1) and Indigo Partnership (MR/R023697/1) awards.

\section{Authors' contributions}

All authors contributed to designing the study, interpreting the results and writing and approving the final manuscript. Bachmann and Grady carried out statistical analyses and wrote the first draft of the manuscript.

\section{Acknowledgements}

The authors thank the clinic nurses and counsellors who participated in the trial; physicians and clinic managers at the study facilities; the North West Provincial Department of Health; and the North West district management. This study is an output of the Programme for improving mental health care (PRIME).

\section{Ethical standards}

The authors assert that all procedures contributing to this work comply with the ethical standards of the relevant national and institutional committees on human experimentation and with the Helsinki Declaration of 1975, as revised in 2008. Ethical approval was obtained from the University of KwaZulu-Natal's Biomedical Research Ethics Committee (reference BFC049/15); the University of Cape Town's Faculty of Health Sciences Human Research Ethics Committee (reference HREC 412/2011) and the Department of Health in the North West Province of South Africa. Participants provided verbal consent for screening and written informed consent before data collection.

\section{References}

1. Academy of Medical Sciences. Multimorbidity: A Priority for Global Health Research. 2018. [cited 2019 Jun 30] Available from: https://acmedsci.ac.uk/policy/policy-projects/multimorbidity.

2. Stubbs B, Vancampfort D, Veronese N, Kahl KG, Mitchell AJ, Lin PY, et al. Depression and physical health multimorbidity: primary data and countrywide meta-analysis of population data from 190593 people across 43 low- and middle-income countries. Psychol Med 2017; 47: 2107-2117.

3. Vancampfort D, Koyanagi A, Hallgren M, Probst M, Stubbs B. The relationship between chronic physical conditions, multimorbidity and anxiety in the general population: A global perspective across 42 countries. Gen Hosp Psych 2017;45: 1-6. 
4. Arokiasamy P, Uttamacharya U, Jain K, Biritwum RB, Yawson AE, Wu F, et al. The impact of multimorbidity on adult physical and mental health in low- and middle-income countries: what does the study on global ageing and adult health (SAGE) reveal? BMC Med 2015;13:178.

5. Moussavi S, Chatterji S, Verdes E, Tandon A, Patel, V, Ustun B. Depression, chronic diseases, and decrements in health: results from the World Health Surveys. Lancet 2007; 370:851-858.

6. Voinov B, Richie WD, Bailey RK. Depression and chronic diseases: it is time for a synergistic mental health and primary care approach. Primary Care Companion For CNS Disorders, 15(2), PCC.12r01468. 2013. Available from: doi:10.4088. PCC.12r01468

7. Scott KM, Lim C1, Al-Hamzawi A, Alonso J, Bruffaerts R, Caldas-de-Almeida JM, et al. Association of mental disorders with subsequent chronic physical conditions: world mental health surveys from 17 countries. JAMA Psych 2016;73:150-158.

8. Jackson CA, Sudlow CLM, Mishra GD. Psychological distress and risk of myocardial infarction and stroke in the 45 and Up Study. Circulation: Cardiovascular Quality Outcomes 2018;11:e004500.

9. Kelley-Moore $\mathrm{J}$ and Ferroaro K. A 3D model of health decline: Disease, disability and depression among black and white older adults. Journal of Health and Social Behaviour 2005;46:376 - 391.

10. Ormel J, Rijsdijk FV, Sullivan M, van Sonderen E, Kempen GI. Temporal and reciprocal relationship between IADL, ADL disability and depressive symptoms in late life. J Gerontol B Psych Sci Soc Sci 2002; 57:338-347.

11. Choi N, Jinseok K, Marti CN, Chen J. Late-life depression and cardiovascular disease burden: examination of reciprocal relationship. Am J Geriatric Psych 2014;22;1522-1529.

12. Mayosi BM, Lawn JE, van Niekerk A, Bradshaw D, Abdool Karim SS, Coovadia HM. Health in South Africa: changes and challenges since 2009. Lancet 2012;380:2029-2043.

13. Mayosi BM, Flisher AJ., Lalloo UG, Sitas, F, Tollman, S, Bradshaw, D. The burden of non-communicable diseases in South Africa. Lancet 2009;374:934-947.

14. Pillay-van Wyk P, Msemburi W, Laubscher R, Dorrington RE, Groenewald P, Glass T, et al. Mortality trends and differentials in South Africa from 1997 to 2012: second National Burden of Disease Study. Lancet Global Health 2016;4:e642-563

15. Herman AA, Stein DJ, Seedat S, Heeringa SG, Moomal H, Williams DR. The South African stress and health (SASH) study: $12-$ month and lifetime prevalence of common mental disorders. S Afr Med J 2009; 99:339-344.

16. Petersen I, Rathod S, Kathree T, Selohilwe O, Bhana A. Risk correlates for physical-mental multimorbidities in South Africa: a cross-sectional study. Epidemiol and Psych Sci 2017:4, 1-9.

17. Statistics South Africa (2018). Community Survey 2016: Profiling socio-economic status and living arrangements of persons with disabilities in South Africa. Report 03-01-23. Pretoria: Statistics South Africa; 2018. Available from: http://cs2016.statssa.gov.za/wp-content/uploads/2018/07/CS-2016-Disability-Report_-03-01-232016.pdf. [cited 2019 Jun 11].

18. Elwell-Sutton T, Folb N, Clark A, Fairall LR, Lund C, Bachmann MO. Socioeconomic position and depression in South African adults with long-term health conditions: a longitudinal study of causal pathways. Epidemiol Psych Sci 2019;28:199-209.

19. Folb N, Lund C, Fairall LR, Timmerman V, Levitt NS, Steyn K, Bachmann MO. Socioeconomic predictors and consequences of depression among primary care attenders with non-communicable diseases in the Western Cape, South Africa: cohort study within a randomised trial. BMC Public Health 2015;15:1194

20. Petersen I, Bhana A, Folb N, Thornicroft G, Zani B, Selohilwe O, et al. Collaborative care for the detection and management of depression among adults with hypertension in South Africa: study protocol for the PRIME-SA randomised control trial. Trials 2018;19,192.

21. Lund C, Tomlinson M, De Silva M, Fekadu A, Shidhaye R, Jordans M, et al. PRIME: a programme to reduce the treatment gap for mental disorders in five low- and middle-income countries. PLoS Med 2012;9(12): e1001359.

22. Bhana, A, Rathod, S D, Selohilwe, O, Kathree, T, \& Petersen, I. The validity of the Patient Health Questionnaire for screening depression in chronic care patients in primary health care in South Africa. BMC Psych 2015; 15, 118.

23. Fairall L, Petersen I, Zani B, Folb N, Georgeu-Pepper D, Selohilwe O, et al. Collaborative care for the detection and management of depression among adults receiving antiretroviral therapy in South Africa: study protocol for the CobALT randomised controlled trial. Trials 2018; 22;19:193.

24. Cornick R , Picken S, Wattrus C, Awotiwon A, Carkeek E, Hannington J, et al. The Practical Approach to Care Kit (PACK) guide: developing a clinical decision support tool to simplify, standardise and strengthen primary healthcare delivery. BMJ Global

Page $14 / 16$ 
Health 2018;3(Supplement 5):e000962

25. Kroenke, K, Spitzer, R. L, \& Williams, JB. The PHQ-9: validity of a brief depression severity measure. J Gen Int Med 2001;16, 606613.

26. World Health Organisation. Measuring health and disability: The manual for WHO Disability Assessment Schedule (WHODAS 2.0). 2010. Available from: https://www.who.int/classifications/icf/whodasii/en/ [cited 2019 Jun 11]

27. Cohen C, Edmondson D, Kronish IM. State of the art review: Depression, stress, anxiety and cardiovascular disease. Am J Hypertension 2015;28, 1295-1302.

28. Holt RIG, Phillips DIW, Jameson KA, Cooper C, Dennison EM, Peveler RC, Hertfordshire Cohort study group (2012). The relationship between depression anxiety and cardiovascular disease: findings from the Hertfordshire Cohort Study. J Affective Disorders 2012;50: 84-90.

29. Kim TJ, von dem Knesebeck $O$ (2018). Income and obesity: what is the direction of the relationship? A systematic review and meta-analysis. BMJ Open 2018;8, e019862.

30. Glymour MM. Using causal diagrams to understand common problems in social epidemiology. In Oakes JM, Kaufman JM, eds, Social Epidemiology. Jossey-Bass: San Francisco. 2006, 393-427.

\section{Figures}

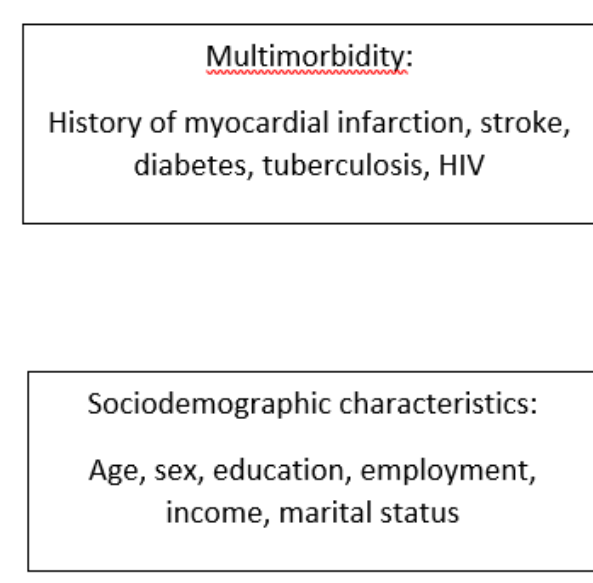

Exogenous variables
I

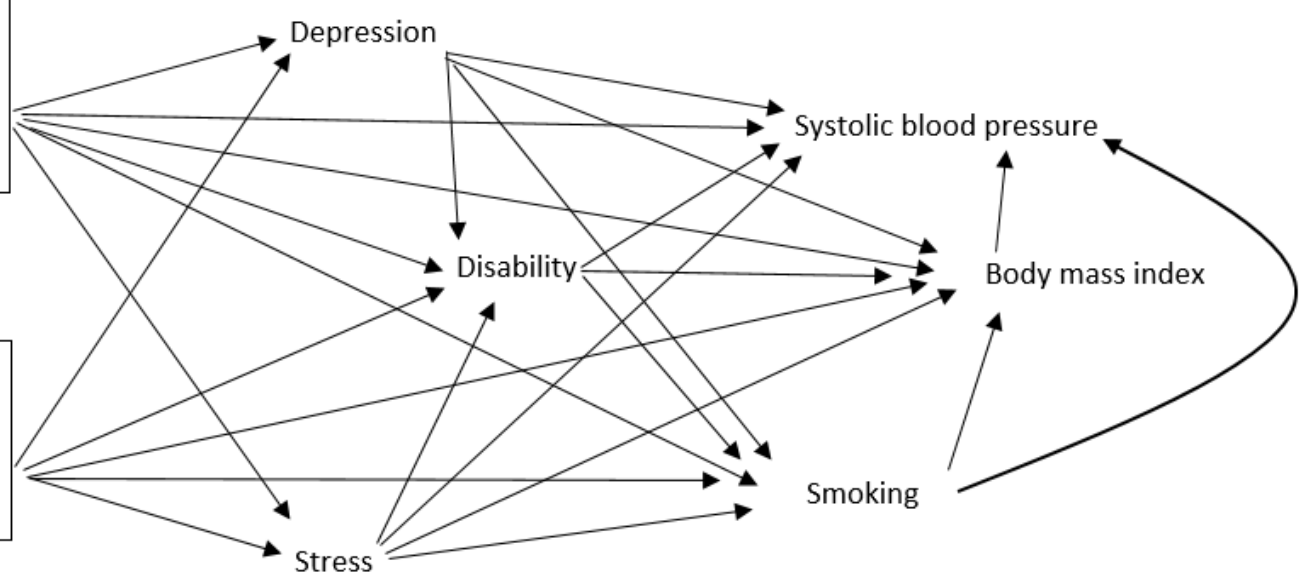

\section{Figure 1}

Structural equation path model for baseline cross sectional data 


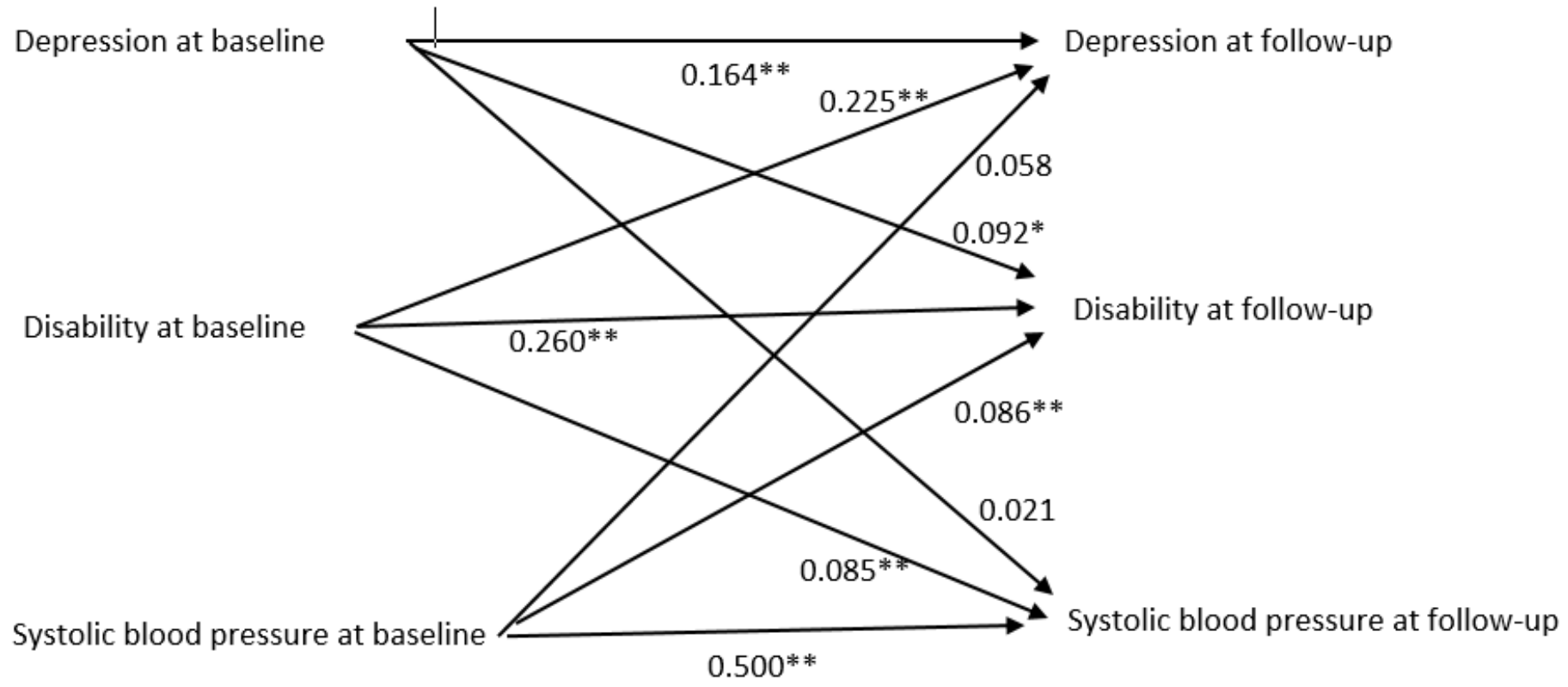

Figure 2

Cross-lagged structural equation model of bidirectional effects over time. All models adjusted for age, sex, marital status, myocardial infarction, tuberculosis, HIV, trial arm and intra-cluster correlation of outcomes. ${ }^{*} \mathrm{P}<0.05,{ }^{\star \star} \mathrm{P}<0.01$

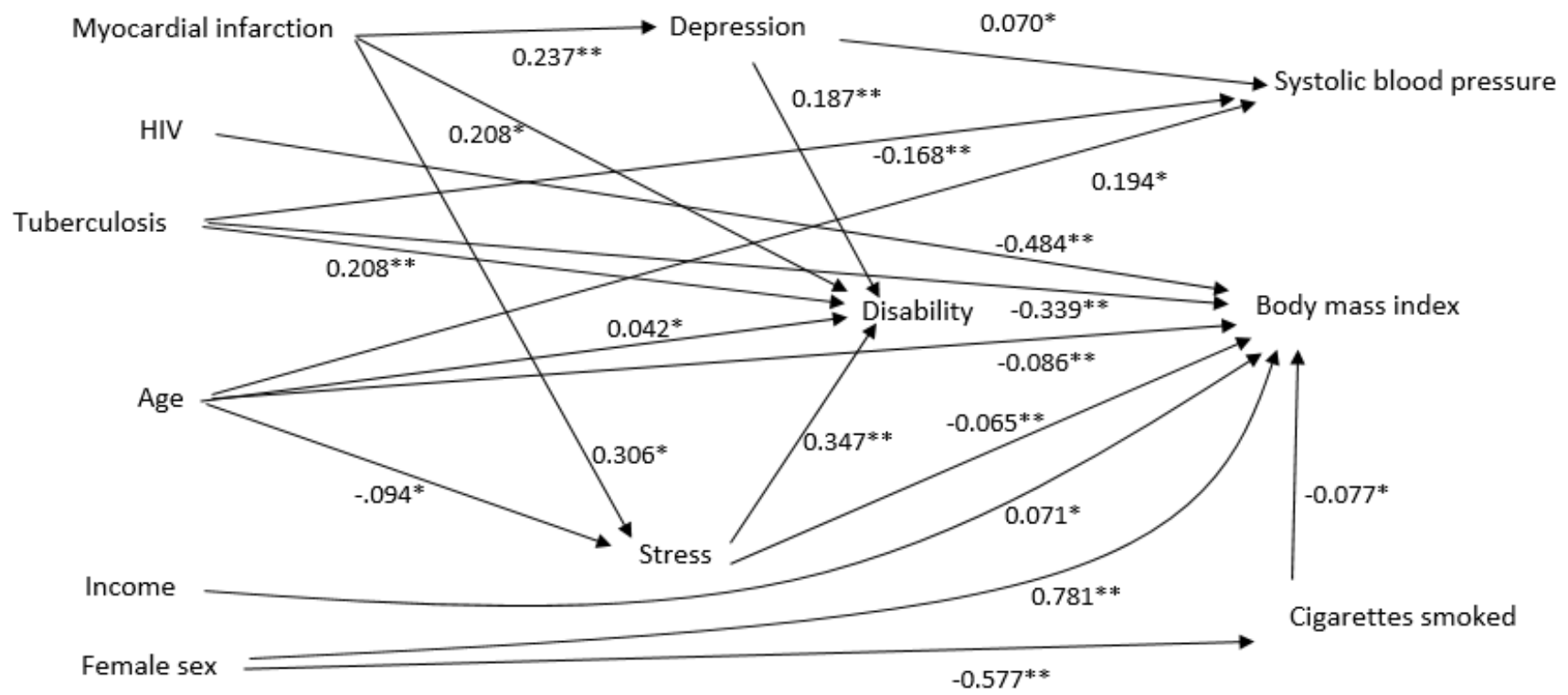

\section{Figure 3}

Statistically significant direct effects on endogenous variables at baseline. Regression coefficients from Table 3. ${ }^{*} P<0.05,{ }^{*} P<0.01$

\section{Supplementary Files}

This is a list of supplementary files associated with this preprint. Click to download.

- Supplementarytables.docx 